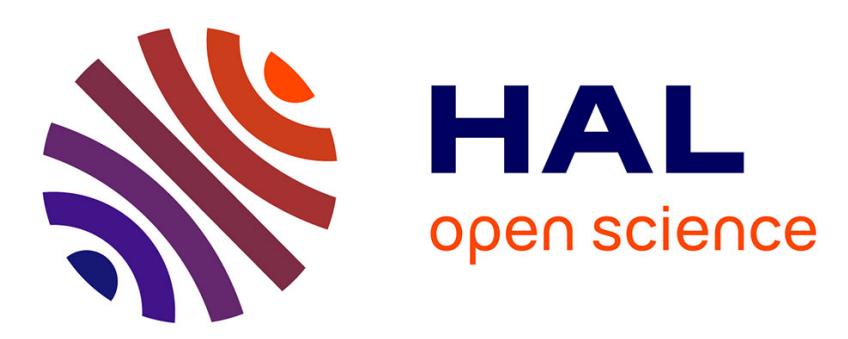

\title{
Customer Relationship Management - Entre injonction à la créativité et fonction de cadrage d'une interaction médiatisée
}

Fabien Bonnet

\section{- To cite this version:}

Fabien Bonnet. Customer Relationship Management - Entre injonction à la créativité et fonction de cadrage d'une interaction médiatisée. Les Enjeux de l'information et de la communication, 2015, 16/3B, pp.111-120. hal-01313161

\section{HAL Id: hal-01313161 \\ https://hal.science/hal-01313161}

Submitted on 19 May 2016

HAL is a multi-disciplinary open access archive for the deposit and dissemination of scientific research documents, whether they are published or not. The documents may come from teaching and research institutions in France or abroad, or from public or private research centers.
L'archive ouverte pluridisciplinaire HAL, est destinée au dépôt et à la diffusion de documents scientifiques de niveau recherche, publiés ou non, émanant des établissements d'enseignement et de recherche français ou étrangers, des laboratoires publics ou privés. 


\section{CUSTOMER RELATIONSHIP MANAGEMENT :}

\section{ENTRE INJONCTION A LA CREATIVITE ET FONCTION DE CADRAGE}

\section{D'UNE INTERACTION MEDIATISEE}

Alors que le marketing, imprégné des organisations et des logiques industrielles avait trouvé en la publicité les ressorts d'un lien formel et unilatéral envers le client, il semble que, sous l'influence des possibilités ouvertes par l'émergence de nouveaux supports et usages médiatiques, ce dernier se soit emparé du champ du social et qu'il ait ainsi opéré un déplacement paradigmatique lui permettant d'afficher des formes de médiation vers une cible désormais présentée comme un auteur impliqué et interprétant à part entière, comme un créateur de sens.

Notre réflexion trouve son origine dans les travaux que nous avons pu mener dans le cadre de notre recherche doctorale (F. Bonnet, 2012), travaux qui nous ont donné l'occasion de questionner la manière dont EDF a cherché à construire sur le long terme son rapport à la clientèle. Nous avons notamment pu mener une analyse à la fois diachronique et synchronique du rapport entretenu par l'ancienne entreprise d'Etat avec son public entre la nationalisation de 1946 et l'introduction, puis le repositionnement de la marque commerciale EDF Bleu Ciel en 2013. Nous avons ainsi pu voir les messages diffusés illustrer tour à tour les différentes dimensions de la structure ternaire du signe mise en évidence par Peirce. En effet, ces messages ont tout d'abord fait majoritairement appel une dimension indicielle, puis, avec l'émergence de la communication institutionnelle, à une dimension iconique, pour finalement affirmer une dimension symbolique appelant des formes de médiation plus indirectes, des conventions de signification plus arbitraires. Ces travaux nous ont permis de conclure à un glissement du discours de l'entreprise et des pratiques de communication de cette dernière vers des médiations symboliques et techniques toujours plus complexes. Par ailleurs, la prise en compte des discours d'accompagnement proposés par les professionnels du secteur nous a conduit à 
insister sur la volonté déclarée de ces derniers de mettre en scène et d'outiller la l'appropriation par les clients des messages et dispositifs complexes proposés. A l'heure des buzz, de la gamification et plus largement de multiples schémas stratégiques visant au développement d'une dimension participative, de l'implication affective, culturelle, des cibles, l'ensemble de ces constats nous a conduit à envisager la créativité des consommateurs indépendamment de toute notion de création, mais en termes plus larges, en termes d'appropriation et donc de coconstruction d'une signification par des organisations et des publics. Une analyse de ces phénomènes à travers les processus de signification qu'ils engagent nous a finalement poussé à questionner les conditions de cette co-construction d'une signification souhaitée par l'entreprise. Ce qui nous semble en jeu quand les formes se brouillent, quand l'appel à l'implication et à la créativité du public se fait plus pressant, c'est en fait la médiation opérée par l'entreprise à travers une pluralité de messages et de supports, et le cas d'EDF plaide selon nous en faveur d'une certaine prudence en matière de création publicitaire.

Qu'en est-il donc des stratégies et des outils déployés par les organisations en faveur de l'engagement du client et faisant appel à une " créativité » de ce dernier ? A quels enjeux ambitionnent-elles de faire face en tentant d'intégrer, voire de s'approprier le champ du social ? En nous livrant ici à une analyse des principes et des démarches mobilisés par les professionnels depuis l'émergence des stratégies dites de « marketing relationnel » (Hetzel, 2004), nous chercherons à montrer que les discours et pratiques marketing actuels mobilisent une créativité très cadrée, tant dans sa définition que dans sa pratique. Nous tenterons enfin de témoigner des apports d'une démarche appréhendant cette mobilisation du paradigme créatif comme une tentative, de la part de professionnels confrontés à d'importantes évolutions de leur métier, de se pourvoir de nouveaux leviers de détection, de gestion et finalement d'action envers un client présenté comme plus émancipé et volatile au plan de ses usages et de la symbolique de sa consommation. 


\section{Développement de la dimension sociale dans les discours et les}

\section{pratiques marketing}

Le marketing serait passé d'un paradigme «transactionnel » à une approche qualifiée de "relationnelle »... Les spécialistes du marketing, qu'ils soient praticiens ou chercheurs, semblent s'accorder depuis quelques années sur le constat d'une réorientation de leurs pratiques professionnelles en faveur d'une Gestion de la Relation Client, en anglais "Customer Relationship Management» (Hetzel, 2004). Au delà d'une transaction commerciale ponctuelle, les outils technologiques et les informations dont bénéficient aujourd'hui les organisations leur permettraient d'entretenir une " relation » avec leurs différents publics. L'émergence de nouveaux outils auraient ainsi permis aux marketers de résoudre un paradoxe bien connu lié à leur volonté de s'adresser individuellement à la foule. Si on en croit un article publié récemment dans l'Harvard Business Review (de Swaan Arons et al., 2015, pp. 3746), les entreprises les plus performantes seraient ainsi celles qui, à travers une évolution de leurs modes d'organisation, parviendraient à exploiter au mieux les données générées par leurs interactions avec les clients pour nourrir cette relation et finalement incarner des valeurs propres à leurs marques.

Dans ce contexte fortement marqué par l'évolution des outils disponibles, les stratégies de communication mises en place par les organisations se révèlent de plus en plus hybrides. En effet, le recours à d'importantes (et coûteuses) bases de données peut permettre la collecte de traces des différentes interactions, traces qui pourront, après exploitation algorithmique, représenter une forme de mémoire de la relation garante de la cohérence de l' " expérience " vécue et perçue par un client dont le comportement et finalement l'identité (Galinon-Mélénec, 2011) sont l'objet de tentatives récurrentes d'analyse et de modélisation.

Ces pratiques marketing se doublent de nombreux discours introduisant un ensemble de notions sensées expliquer les évolutions en cours ou à venir mais dont Sylvie P. Alemanno (P. Alemanno, 2014) a bien montré la dimension essentiellement promotionnelle, voire performative. La communication d'entreprise serait donc " multicanal » (avec ou sans « e »?), " crossmedia », inspirée par un "marketing $360^{\circ}$ » garant de la cohérence, de la persistance, de l'expérience vécue à travers différents médias, de la boutique au site web, du centre d'appel à l'application 
mobile... Outillée de manière efficiente, la transaction devenue relation permettrait un échange quasi informel entre entreprise et client, une " conversation » dont la figure du Community Manager semble être l'incarnation contemporaine (Galibert, 2014). Cependant, la définition encore floue de la place et du rôle de ce dernier au sein des organisations atteste selon nous d'une tension inhérente à cette relation décrite comme spontanée mais stratégiquement déterminée. Les prouesses les plus médiatisées de ces « $\mathrm{CM}$ » ne sont elles pas issues de situations où ils doivent faire le grand écart entre la parole institutionnelle et une forme parfois malvenue de spontanéité du public illustrée par la figure du "troll ». Leurs succès seraient donc attribués à leur capacité à assurer seuls la médiation entre entreprise et grand public, finalement à incarner la «convergence » présentée comme caractéristique du Customer Relationship Management.

Dans ce contexte, la configuration et la mise en scène d'une expérience cohérente pour le client s'appuient à la fois sur une diversification des supports employés et des stratégies éditoriales, mais aussi sur une évolution de la place et du rôle des fonctions marketing et communication au sein des entreprises. Portée dans les petites structures par la professionnalisation progressive d'une fonction communication à dimension stratégique, que ce soit en interne (service ou direction communication) ou en externe (recours à une agence), cette cohérence conduit les organisations de plus grande taille à revoir leur organigramme. En effet, l'émergence conjointe du Customer Relationship Management et du management par projet en lieu et place d'une hiérarchie dédiée à la seule vente d'un produit ou service pourraient conduire à une affirmation du marketing et de la communication comme fonctions en charge d'une médiation interne aux projets, ce que défendent de nombreux praticiens (Joshi \& Giménez, 2015). Sous des intitulés tels que «Chef de projet opérationnel » ou « Consultant » (en agence), ces fonctions intégratrices sont en effet susceptibles d'acquérir un statut qui rappelle celui du design industriel sensé permettre, à travers une approche transversale, une prise en compte des usages dans la conception des produits. Nous remarquerons cependant que des travaux menés dans le champ de la communication (Lépine, Martin-Juchat, Millet-Fourrier, 2014) des organisations tendent pourtant à montrer une perte de capacité de la fonction communication à assurer le dialogue stratégique dans les organisations, au profit d'un rôle de commanditaire joué par la seule fonction marketing. Ce qui peut 
alors apparaître comme un écart de points de vue nous semble en fait très représentatif d'une question de nature managériale, celle de la répartition des compétences et des ressources entre les fonctions communication et marketing. Ces deux secteurs d'activité investissent en effet le même objet qu'est la médiation entre l'organisation et ses publics, qu'ils soient internes ou externes. Les questions traitées, les démarches et les outils mis en place ainsi que la temporalité des projets diffèrent cependant largement. Nous plaiderons davantage en faveur d'une approche intégrative de ces questions de communication, en faveur d'une approche communicationnelle appréhendant les dispositifs et stratégies mobilisés sous l'angle des processus de signification qu'ils outillent.

Cristallisant aujourd'hui une forme de médiation interne aux organisations, garante d'un échange avec les publics et portée par une diversité d'acteurs et de supports, la communication entrepreneuriale voit donc son statut évoluer. Si Roland Barthes et Jean Baudrillard avaient déjà insisté en leur temps sur le statut mythique de certains produits de consommation et de leur promotion, la question du statut sociétal des discours commerciaux reste en effet d'actualité, comme en témoignent les travaux de Karine Berthelot-Guiet. En évoquant avec Valérie Patrin-Leclère et Caroline Marti de Montety (Berthelot-Guiet et al. 2013) un double processus de dépublicitarisation / hyperpublicitarisation, celle-ci met bien en évidence la capacité d'hybridation de la communication entrepreneuriale, sa capacité à investir de nouveaux formats, à adopter de nouvelles stratégies éditoriales et finalement à renoncer en partie à une forme ouvertement publicitaire. Cependant, l'émergence de ces pratiques, vue par les professionnels comme celle d'un «Brand Content» (Jamet, 2013), serait paradoxalement à l'origine d'une hyperpublicitarisation, d'un décloisonnement de la communication publicitaire qui, omniprésente et quasi indiscernable parmi les productions médiatiques, se trouverait banalisée, infraordinarisée. Ce marketing devenu « relationnel » et traduit en stratégies multimédiatiques nous rappelle la notion de "relations publiques généralisées » introduite par Bernard Miège (Miège, 2007). Selon ses promoteurs, la sophistication récente des stratégies et dispositifs marketing en aurait fait une forme de médiation culturelle et sociale, une médiation pensée et mise en œuvre par des organisations attirées par le potentiel commercial d'une hypothétique « Brand Culture » (Bô \& Guével, 2013). 


\section{Affirmation d'une approche gestionnaire plutôt que culturelle : vers}

\section{une relation gérée?}

Du point de vue de la recherche en Sciences Humaines et Sociales, et a fortiori en Sciences de l'Information et de la Communication, la mobilisation de la notion de culture pour caractériser un horizon des stratégies marketing contemporaines pose cependant problème. On peut à ce titre reprendre les réserves exprimées par Yves Jeanneret face à " des acteurs du monde marchand " qui, selon lui, " après avoir réussi à faire admettre ce terme de communication comme un euphémisme pour désigner la réclame (agences de communication, campagne de communication, etc.) (...) poussent l'avantage et en viennent à prétendre être des médiateurs de culture, voire à se faire médias " (in. Berthelot-Guiet et al., 2014, p. 7). Mais comment aborder ces pratiques commerciales et ces contenus médiatiques sans tomber dans le procès d'intention, sans occulter la complexité des liens entre médiation marchande, culture et champ du social ?

La dimension stratégique de la démarche marketing est en effet synonyme d'intentionnalité, mais d'une intentionnalité managériale que l'on pourra aborder avec Christian Le Moënne sous l'angle soit d'une structuration organisationnelle visant l'efficience dans la réalisation d'objectifs, soit sous celui plus critique d'une "idéologie managériale de la rationalité des méthodes organisationnelles" (in P. Alemanno, 2014, p. 17). Avec l'essor du CRM, nous assistons à une complexification du dispositif de communication mis en place par les organisations pour outiller et mettre en scène une médiation commerciale. Ce dispositif, dans ses dimensions technique, humaine, procédurale, médiatique, ne peut bien sûr être considéré comme neutre du point de vue des processus de signification qu'il mobilise et de la nature de l'interaction qu'il implique. Si tout dispositif peut être compris en termes de «stratégies de rapport de force supportant des types de savoir et supportés par eux » (Foucault, 1994 cit. Agamben, 2007, p. 10), cette complexification s'avère technologiquement, sémiotiquement, culturellement lourde. Elle semble même en mesure de renforcer l'impact de ce qu'Etienne Candel définit comme un «cadrage éditorial » de la communication médiatisée. Les échanges entre entreprises et clients se trouvent en effet conditionnés par les contraintes inhérentes aux nombreux dispositifs mobilisés, aux usages de ces derniers, aux attentes de leurs concepteurs. 
A titre d'exemple, les $\mathrm{API}^{1}$ proposées aux développeurs par des entreprises comme Apple et Google conditionnent l'ergonomie des applications et représentent ainsi un cadre pour l'interaction médiatisée par les terminaux mobiles. Les interactions autorisées ou non par les différents réseaux sociaux ainsi que les chartes et scripts utilisés par les téléconseillers en centres d'appel représentent autant de contraintes pour cette « relation » dont les professionnels du marketing se réclament aujourd'hui.

L'interaction avec le client développée dans le cadre de stratégies de CRM se trouve donc multi-cadrée, tant d'un point de vue téléologique, stratégique, que de celui des contraintes techniques et éditoriales liées à la diversité des supports et outils mobilisés. Alors que la "relation client» ainsi gérée s'affirme comme un critère concurrentiel majeur abordé en termes de «qualité », le principe selon lequel la fidélisation d'un client coûterait moins cher qu'une stratégie de conquête fait aujourd'hui partie des fondamentaux de l'enseignement en marketing. II s'agit alors de configurer un dispositif, un enchaînement d'étapes, de tâches visant à satisfaire et fidéliser la clientèle tout en assurant des gains de productivité. Cette configuration passe entre autres par la mise en œuvre et l'outillage d'une procédure finalement présentée en termes d'« optimisation du processus client» (Peelen et al., 2009). Malgré les discours d'accompagnement massivement diffusés par les promoteurs de ce type de démarche, le recours aux notions d'optimisation et de processus, elles même héritées de l'ingénierie et du management industriel, apparaît alors comme symptomatique d'une approche de la relation bien plus technique et gestionnaire que sociale, psychologique et culturelle.

Mais plutôt que de chercher à distinguer ce qui, dans une démarche industrielle caractérisée par son utilitarisme, relèverait du pragmatisme ou de l' "idéologie managériale " évoqués par Le Moënne, nous avons eu l'occasion d'insister sur la nécessité de distinguer la « relation client », stratégique et outillée, de la "relation au client » associée à une médiation sociale et culturelle (F. Bonnet, 2012). Dans cette perspective, le CRM, en mobilisant le paradigme relationnel pour promouvoir et légitimer des pratiques commerciales, apparaît avant tout comme révélateur d'une tension majeure entre intentionnalité des stratégies de communication outillées selon des logiques industrielles et appel à l'implication de chacun sous couvert d'une

\footnotetext{
${ }^{1}$ Application Programming Interface, Interface de programmation
} 
médiation entrepreneuriale. Finalement, plutôt que de chercher à évaluer la dimension "relationnelle » de l'échange entre organisations et public, ce qui revient finalement à adopter une posture assez normative, c'est l'appel implicite ou explicite à l'implication de ces publics dans la consommation qu'il nous semble nécessaire de questionner pour comprendre les évolutions récentes des pratiques marketing.

\section{Marketing et créativité : une injonction paradoxale?}

Si on considère ces stratégies CRM d'un point de vue psychologique, la supposée implication du client permettant aux organisations de filer la « métaphore sociale » ne peut être réduite à la seule question de la fréquence des interactions. En effet, au delà d'une simple proximité pensée en termes topographiques, d'une participation désincarnée, celle-ci implique l'attribution, par le public, d'une signification à l'organisation, à ses activités et, plus globalement, à la consommation.

Cette réflexion trouve un écho particulièrement fort dans deux contributions récentes proposées respectivement par Françoise Bernard et Fabienne Martin-Juchat, lesquelles insistent sur deux dimensions complémentaires des processus de signification outillés par le numérique. Françoise Bernard (in. P. Alemanno, 2014, p. 45-58) en propose une lecture en termes d' " engagement rationalisant » des acteurs de la communication organisationnelle quand Fabienne Martin-Juchat (in. P. Alemanno, 2014, p. 59-68) met l'accent sur la « valeur ajoutée affective » dégagée par ces derniers et mobilisée en tant que critère pour une interprétation et une construction du réel. Dans les deux cas, on comprend que les stratégies de communication mises en place par les organisations se trouvent confrontées à des publics en recherche de sens, loin de l'image caricaturale d'une passivité face à la « réclame ».

En tant qu'attribution de signification, l'implication évoquée, souhaitée et revendiquée par les professionnels du marketing revêt donc une dimension représentationnelle et axiologique, laquelle nécessite selon nous d'être comprise dans sa complexité, en lien avec son contexte d'émergence. Nous nous inspirons ainsi des travaux de Sandra Jovchelovitch (Jovchelovitch, 2007) pour l'aborder en termes de « processus représentationnel », de processus de signification. Cette approche à la fois psychosociale et processuelle nous semble en effet en mesure de permettre une 
remise en question précise de nombreux termes gestionnaires tendant à réifier des dynamiques culturelles complexes en en dissimulant les enjeux. La tendance des professionnels du marketing à évoquer une «identité d'entreprise », en laquelle Julien Tassel (Tassel, 2013) reconnaît une forme de réification dissimulant le poids d'une construction symbolique, nous semble par exemple justifier ce type d'approche. En effet, ce dernier met bien en évidence des pratiques intervenant à différents niveaux d'un processus de signification et visant à condenser un certain nombre de caractéristiques organisationnelles pour incarner, naturaliser et finalement porter une stratégie managériale.

De la même manière, la « créativité » des clients auquel cet article est consacré peut selon nous être envisagée comme une notion réifiante issue des pratiques marketing et portée par les discours publicitaires. En effet, dans le cadre de notre approche psychosociale et communicationnelle, il nous semble intéressant de l'appréhender avant tout comme une appropriation, par les publics, d'une offre proposée par les organisations via différents dispositifs. Définie comme un bricolage de sens, en termes de construction et d'attribution de signification, cette créativité nécessite une implication vis-à-vis de l'organisation concernée, implication qui va bien au delà de la « relation » technicisée revendiquée par les Marketeurs. Elle passe en effet par une reconnaissance de l'identité donnée à voir par l'organisation, par une reconnaissance de la part de publics qui établissent un lien de sens entre message perçu et représentations préexistantes. Dans un contexte médiatique marqué par l'hyperpublicitarisation évoquée plus haut, par une surcharge informationnelle couplée à une remise en cause du statut des médias traditionnels, de nombreux professionnels voient dans le développement de stratégies de marques une solution efficace pour susciter et soutenir cette reconnaissance. A l'intersection des problématiques de notoriété et de réputation, ces stratégies s'appuient sur une tentative de cristallisation des signes, représentations sociales et valeurs associées à l'organisation en vue d'en faire une composante à part entière de l'offre de service formulée par cette dernière, offre à la fois technique et culturelle, sensible et symbolique, sensible au plan de la perception, de l'interprétation par les clients, symbolique en termes de formes et de messages mobilisés. C'est dans ce travail d'ingénierie symbolique, dans cette attention portée au renforcement de la dimension symbolique des offres qu'il faut selon nous voir une injonction à l'implication du client. 
En effet, la segmentation des marchés se traduit aujourd'hui par une multiplication des positionnements marketing suggérant un statut accordé au public et notamment son implication qui n'est plus celle d'un client supposé rationnel confronté à un produit mais au contraire celle d'un individu interprétant, s'appropriant des propositions symboliques complexes diffusées par des organisations devenues acteurs «culturels ». Cependant, en tant que constructions symboliques diffusées à des fins de persuasion, les marques peuvent bien sûr être lues sous l'angle d'une euphémisation, d'une tentative d'ellipse de toute divergence liée à cette appropriation, à cette interprétation. Au sein des stratégies marketing contemporaines, l'intentionnalité managériale se traduirait donc, comme le souligne Marine Allein (Allein, 2015), par un appel à une implication cadrée qui viserait paradoxalement à contourner la difficulté d'une relation à l'altérité.

Selon nous, il ne s'agit dans pas tant de savoir si le client est réellement impliqué dans les activités de l'organisation, que d'insister sur le processus de mise en scène de cette implication, sur les démarches et les formes mobilisées pour la suggérer et la soutenir. Or, cette construction symbolique de l'organisation passe aujourd'hui par un soin particulier accordé à une certaine dynamique de la mise en scène, à une forme de prise de distance par rapport à une parole entrepreneuriale qui ne serait que déclaration publicitaire, que réclame décontextualisée et rétrograde. Ce rejet apparent d'une approche transmissive de la communication se traduit par la constitution et la diffusion de récits, ces derniers mettant aussi bien en scène l'organisation elle-même que ses publics. Dans cette perspective, les discours accompagnant la mise en place de stratégies et de dispositifs CRM peuvent finalement être appréhendés à l'aune d'une mise en récit de la relation au client, d'une mise en scène du rapport à un client figuré comme actif, non plus spectateur de la publicité mais acteur d'une relation avec les organisations. De la même manière que le « créatif » en agence de communication n'est plus représenté comme salarié investi dans une tâche commerciale mais comme créateur, nous considérons l'individu créatif comme une figure centrale de cette mise en scène, de ce storytelling racontant l'investissement du client dans sa consommation. La mise en scène de cette créativité du public, créativité cadrée par les dispositifs mobilisés, apparaît alors comme une forme rhétorique caractéristique des stratégies marketing contemporaines et visant à éviter que l'évocation d'une relation au client ne rende 
possible une divergence d'interprétation, une altérité dans le processus de signification conduisant à la co-construction d'une signification pour la marque.

\section{La curation créative comme consensus?}

Dans ces conditions, l'importance accordée au paradigme créatif dans les discours sur la consommation ne peut selon nous être réduite à une question de créativité des publicitaires ou même des clients dans leurs pratiques. Son affirmation comme thématique prééminente des discours marketing, voire comme revendication, gagne en effet à être appréhendée en lien avec son contexte d'émergence, et notamment avec une évolution importante des pratiques médiatiques en lien avec la consommation.

Si la créativité n'est pas nécessairement la première qualité attendue d'un commerçant, la capacité d'un communicant à bricoler, au sens de Lévi-Strauss, avec la diversité des cadres éditoriaux dans un contexte de dépublicitarisation représente aujourd'hui un élément essentiel de sa professionnalité, à la fois de sa compétence et de son mode de socialisation. A l'heure de stratégies marketing dites « relationnelles », revendiquant et outillant une interaction plus riche avec les clients, voire une relation au sens social et culturel du terme, nous abordons cette évolution à l'aune d'une mise en scène, par les organisations, de l'appropriation par les publics de leur offre technique et symbolique. On peut évoquer à titre d'exemple le succès de nouvelles formes de placement de produits dans le cadre d'émissions consacrées à la décoration, émissions mettant aussi bien en scène le produit lui-même que la posture des cibles face aux messages proposés par les acteurs du marché. Que ce soit dans ce type de productions médiatiques ou dans les énoncés d'accompagnement explicitement produits par les professionnels du marketing, la créativité s'affirme comme un thème central et récurrent d'une mise en scène de la conception, de la production et de la circulation des discours marketing eux-mêmes. La dimension « relationnelle » des stratégies marketing contemporaines se traduirait donc par l'émergence d'un discours sur l'appropriation des messages par les publics et finalement par la revendication d'une dimension réflexive dans les pratiques marketing. 
En mettant ainsi en scène leur activité sous un angle qui rappelle la démarche design, les professionnels du marketing donnent à voir un pan de leur activité d'ingénierie symbolique. Le marketing relationnel en contexte de dépublicitarisation, dans la mesure où il tiendrait compte de la perception des publics, marquerait la fin d'une crainte des procès en manipulation et acterait une certaine banalisation des discours commerciaux comme médiation. Face à cette évolution des pratiques médiatiques impliquant aussi bien les organisations que leurs publics, il serait finalement possible de s'interroger quant au potentiel renforcement d'une forme de « curation créative » dans laquelle la thématique commerciale joue un rôle central. On peut à ce titre évoquer la plate-forme sociale Pinterest qui, si elle n'est pas autant utilisée que Facebook et Twitter, représente aujourd'hui l'une des plus importantes capitalisations sur son marché (Eveno, 2015), notamment du fait de son potentiel en termes de mise en scène d'une certaine créativité des marques et de leurs publics. $A$ l'intersection de deux visions de la créativité comme appropriation originale d'un objet (re-présentation) et comme territoire de communication relatif à cet objet pour les organisations (re-production), la curation créative consisterait en une élaboration collective de listes de suggestions sur la base d'informations fournies par les diverses parties prenantes de la consommation. Elle pourrait représenter une forme de consensus entre liberté d'expression pour les organisations et liberté d'interprétation pour les cibles.

Cependant, la mise en scène médiatique de la capacité de jugement des individus face aux propositions commerciales se trouve selon nous en contradiction avec la recherche de maîtrise qui caractérise la démarche managériale. De la même manière que la relation client ne peut être pleinement assimilée à une relation sociale pourtant revendiquée par les entreprises, les stratégies marketing cherchant à suggérer l'implication des cibles peineront selon nous à faire vivre une créativité qui nécessiterait avant tout une liberté d'interprétation en contradiction avec les pratiques de cadrage propres à la communication institutionnelle et avec la notion même de stratégie. Quel statut accordé à la créativité des clients sur le marché de la musique en streaming ? Les pratiques de curation consistant à constituer des listes à l'intérieur même du cadre logiciel et commercial proposé par les plates formes peuvent elles être considérées comme autant de signes d'une véritable médiation entre individu et organisation. De ce point de vue, l'hypothèse d'une curation créative 
comme médiation culturelle fait selon nous l'impasse sur le cadrage stratégique et éditorial opéré par les différents acteurs du marché publicitaire, qu'il s'agisse des annonceurs eux-mêmes ou des concepteurs et promoteurs des médias/canaux actuellement en développement. En tant que mise en scène outillée d'une mobilisation de la créativité dans la consommation, cette curation apparaît alors comme un prolongement de l'évolution des stratégies de communication entrepreneuriale vers les relations publiques. 


\section{Bibliographie}

\section{Travaux de recherche}

Agamben, Giorgio (2007), Qu'est-ce qu'un dispositif ?, Paris: Rivages poche.

Allein, Marine (2015), "L'éthique travaillée par la communication managériale", communication lors de la journée d'études L'éthique dans les pratiques professionnelles de l'information et de la communication, 18/03/15, Celsa (Université Paris-Sorbonne), disponible en ligne : www.gripic.fr

Berthelot-Guiet, Karine ; Marti de Montety, Caroline ; Patrin-Leclère, Valérie (2013), "Entre dépublicitarisation et hyperpublicitarisation, une théorie des métamorphoses du publicitaire", Semen, n³6, pp. 53-68.

Berthelot-Guiet, Karine ; Marti de Montety, Caroline ; Patrin-Leclère, Valérie (2014), La fin de la publicité ?: Tours et contours de la dépublicitarisation, Lormont: Editions Le Bord de l'eau.

Bonnet, Fabien (2012), Evolutions sociétales et mutations de la relation client - Une approche communicationnelle de la relation au client développée par EDF, thèse de doctorat en Sciences de l'Information et de la Communication sous la direction de Nicole D’Almeida, Celsa - Université Paris IV Sorbonne.

Bonnet, Jacques ; Bonnet, Rosette ; Gramaccia, Gino (2011), Management et communication : mutations, emprunts et résonances, Paris : I'Harmattan.

Foucault, Michel (1994), Dits et Ecrits, 1954-1988. Tome III: 1976-1979. Paris : Editions Gallimard.

Galibert, Olivier (2014), Pour une approche communicationnelle du community management, mémoire d'habilitation à diriger les recherches, Université de Bourgogne, Dijon.

Galinon-Mélénec, Béatrice (2011), L’Homme trace. Perspectives anthropologiques des traces contemporaines, Paris : CNRS Editions.

Jovchelovitch, Sandra (2007), "La fonction symbolique et la construction des représentations : la dynamique communicationnelle Ego/Alter/Objet", Hermes, n41. 
Lépine, Valérie (dir.) ; Martin-Juchat, Fabienne (dir.) ; Millet-Fourrier, Christelle (dir.) (2014), Acteurs de la communication des entreprises et organisations: Pratiques et perspectives, Grenoble : PUG.

Miège, Bernard (2007), La Société conquise par la communication: Tome 3, Les Tic entre innovation technique et ancrage social, Grenoble : PUG.

P. Alemanno, Sylvie (Coord.) (2014), Communication organisationnelle, management et numérique, Paris : Editions L'Harmattan.

Tassel, Julien (2013), "L’identité d'entreprise - Réponse du management à l'accélération sociale", Communication, $n^{\circ} 31 / 1$, disponible en ligne http://communication.revues.org/3906

\section{Publications professionnelles et discours d'accompagnement}

Bô, Daniel ; Guével, Mathieu (2013), Brand culture : développer le potentiel culturel des marques, Paris: Dunod.

Eveno, Anne (2015), "Pinterest valorisée 11 milliards de dollars", Le Monde.fr, 17/03/15, disponible en ligne, www.lemonde.fr/economie/article/2015/03/17/pinterestvalorisee-11-milliards-de-dollars_4594860_3234.html

Peelen, Ed ; Jallat, Frédéric ; Stevens, Eric ; Volle, Pierre (2009), Gestion de la relation client. Paris : Pearson Education.

Hetzel, Patrick (2004), Le marketing relationnel, Paris : Presses universitaires de France.

Jamet, Thomas (2013), Les nouveaux défis du Brand Content. Paris : Pearson.

Joshi, Aditya ; Giménez, Frank (2015), "La prise de décision au coeur du marketing", Harvard Business Review France, n7, pp. 49-55.

De Swaan Arons, Marc ; Van den Driest, Frank ; Weed, Keith (2015), "L'ultime machine marketing", Harvard Business Review France, n7, pp. 37-46. 